\title{
Basketball Player's Explosive Force Training Skills
}

\author{
Yiming Zou \\ College of Aviation Security, Civil Aviation Flight University of China, Guanghan, 618307, China
}

Keywords: Basketball. Player. Explosive force. Trailing skills

\begin{abstract}
The explosive force is one of comprehensive abilities combining strength and speed, and it gets the comprehensive characteristics of strength and speed. This paper, based on the distinct characteristics of basketball sports and the factors influencing the basketball player's explosive force, discusses the main physical basis, training contents, methods and means, load intensity and other related contents of the basketball player's explosive force.
\end{abstract}

\section{Introduction}

Modern basketball sports is a sports event with very high intensity, speed and rivalry, and a basketball player has to move continuously and rapidly, jump continually, and strive for the ball positively in a long competition time, needing to complete various skilled movements repeatedly under extremely fierce competition. Therefore, the basketball player needs to bear very heavy load, and it is indeed physically exhausting. By studying the basketball player's explosive force training skills, the author discusses the significance of explosive force in basketball player's training, so as to provide strong theoretical foundation and quantitative indicators for comprehensively promoting the basketball player's explosive force training level and further for promoting the basketball player's performance by use of the training skills in a more scientific way.

\section{Basic factors influencing the explosive force}

The so-called explosive force actually means the energy to give play to the maximum force of muscle in the shortest second. The basic factors influencing the explosive force are the exerciser's muscle contraction speed and the maximum force of muscle contraction. Therefore, to improve the explosive force, it is necessary to greatly promote the maximum force and the muscle contraction speed during the exerciser's muscle contraction process. The muscle contraction speed is generally affected by type of muscle fiber, and the type of muscle fiber is genetic, so it is hard to get improved. Therefore, the important way of improving the explosive force is to develop the maximum force as much as possible, and the development of maximum force mainly depends on physiological cross-section of muscle and the muscle coordination.

\section{Main skills of basketball player's explosive force training}

\section{Training skills based on body parts}

\section{Lower limb's explosive force training skills}

The basketball player may use barbell and other tools to conduct explosive force training, the training methods mainly include deep squat, half squat, dumbbell squat, and forward lunge, etc., and the intensity of training is $50 \%$ to $70 \%$ of the maximum load; it is required to do the movements (six to eight sets of movements), repeat each set of movements for three to five times, and finish each movement as quick as possible, with a break of two to three minutes. The skilled movement of each training method should be correct and quick, and concentrated force can intensify the explosive force exerting way. In addition, various jump trainings for overcoming the self weight are necessary, such as standing triple jump and 30m split leap and double-roll rope skipping, you are required to do each set of movements for ten to fifteen times. In particular, double-roll rope skipping is very effective to develop the lower limb's explosive force. It raises a very high requirement for the basketball player to 
train the lower limb's explosive force, and it is effective to develop the explosive force of shank, foot arch, ankle, and sole, and also the posture in the air after jumping has a very high requirement for the explosive force of the waist and belly, which can develop the explosive force of such parts as waist and belly. It is very effective to develop the basketball player's explosive force by frequently changing the various training methods above and comprehensively using multiple methods and the training can be finished within five to ten seconds by designing the most reasonable speed load and strength load.

First, a load of $90 \%$ to $30 \%$ of the exerciser's strength: first complete two sets of movement training by use of the weight which is $90 \%$ of the maximum strength and then three sets of movement training by use of the weight which is $30 \%$ of the maximum strength, repeat six to eight times for each set of movements, and finish at the fastest speed. It is required to relax the muscles between each repeated practice, take a break of three to four minutes between each set and four to six minutes before changing the load. It is recommended to arrange two to three practice units in each training course, with a break of eight to ten minutes between each unit.

Second, two sets of jumps with kettle-bell: jump for six to eight times in each set, after a rest of three to four minutes, carry out a jumping training with the maximum strength, for example, switch leap with two legs in place for eight times, two sets, and five to six times in each set; the whole set of training shall be repeated for two to three times, with a pause time of six to eight minutes.

Third, a weight which is $80 \%$ to $85 \%$ of the maximum strength: squat with barbell, with at last two to three times in each set and totaling two sets, after a rest of three to four minutes, have two to three sets of squat jump training with kettle-bell, with four to six times in each set; the whole set of training shall be repeated for two to three times, with a pause time of six to eight minutes.

Fourth, a weight which is $90 \%$ of the maximum strength: have two sets of training of squatting by shouldering barbell, eight times in each set, and drop jump for six to eight times, totaling two sets. The rest time between squatting with barbell and jumping is two to four minutes, and the rest time between jumping practices is four to six minutes. Repeat the above combination for two times in each training class, with a break of eight to ten minutes.

Fifth, carry out five sets of training practices, with $90 \%$ of the maximum weight horizontally, including the maximum weight of three times up to 95\%, the maximum weight of one time reaching $97 \%$ and the maximum weight of one time reaching $97 \%$, and take a break of three to four minutes in each set. Relaxation training is suggested. Once the basketball player thinks that he may fail after finishing the last set when using such method for training, it is possible to eliminate the last set, then after a break of six to eight minutes, start anew, but the training with the maximum load of $100 \%$ cannot be eliminated.

\section{The whole body's explosive force training skills}

The whole body's explosive force training skills mainly include continuous quick turning over, stretching, snatching and high shifting, and the movement technology link covers the leg's force of pedaling the ground, knee extending, hip stretching and erecting as well as other various movements. The abovementioned movements are not only able to mobilize the most body to participate in force exerting, but also demanding on the coordination of body's explosive force. The intensity of training is up to $70 \%$ to $85 \%$ of the maximum load. Six to ten sets of training are required, with four to six times each set, with a break of fifteen seconds. The training speed should be rapid, and the explosive force shall be exerted intensively. In addition, it is required to strictly follow various indicators in training.

\section{Explosive force training skills of shoulder girdle, upper limb and wrist}

When carrying out weight training exercise, the basketball player may have the strength training of lying triceps extension, press by sitting, bench press and weight-bearing push-up, with the intensity of training reaching $40 \%$ to $60 \%$ of the maximum load; two to three sets of training are required, with eight to twelve times each set, and the time of break is two minutes. It is required to be quick in this training. So the basketball player should make every effort to exercise. In particular, it is best to pass a solid ball with two hands or shoot at the basket with solid ball. Based on the skilled movements of different basketball players, the difficulty of exercise can be intensified. The 
abovementioned training means are quite close to special skilled movements in basketball competition, which can not only develop the explosive force but also consolidate and improve the skilled movement ability of the basketball player.

\section{Training skills based on form of exercise}

\section{Weight-bearing exercise skills}

Currently, the tendency of large-load exercise method at home and abroad is to actively develop the explosive force, that is, to take an exercise with $85 \%$ load of the personal maximum strength. The simplest and ordinary measuring method of the maximum strength is decided by the maximum weight raised in one time. For example, as for the measurement of maximum strength of weight-bearing squat, it is subject to the optimal strength that the basketball player can squat in one time after bending his knees to half-squat position, with a barbell on the shoulders.

\section{Super-equal-length exercise skills}

A proper drop jump of height is the most effective way to realize super-equal-length explosive force development. Once the load is appropriate, it means that the drop jump is also suitable, and then the lower limb's musculus extensor can be extended reasonably. By stretching the exerciser's muscle reasonably, the effect reflected by muscle tension can be enhanced, and besides, the elasticity component possessed by the stretched muscle reserves quietly flexible potential energy and the energy is released and utilized in the shortened process, therefore properly improving the exerciser's jumping height and distance. In the current exercise of drop jump, what is different is that a proper height should be selected for drop jump development, so as to form greater explosive force.

\section{Substantial exercise skills}

When in weight-bearing exercise, the exercise of movement exceeding the maximum movement range should be included. For example, to make a choice from several similar exercise items, it is necessary to select one exercise with a fairly big distance. After the basketball players repeat the exercise of correct movements for times, they may take a break when their muscle is under fairly sufficient contraction status, to make the muscle under equal-length status, and the maximum strength of muscle under static contraction status can be gained more.

\section{Progressive exercise skills}

The skill of progressive exercise is to use the method of increasing resistance gradually so as to make sure the load weight and the movement speed both get a stable improvement. The load weight applied in each set is subject to the weight of six weights that can be raised exactly. Once the movement speed can reach the maximum degree, the load weight can be added to the weight that can be completed only in six times, and so forth.

\section{Principles of basketball player's explosive force training}

The basketball player's explosive force training shall follow the principle of specificity. As far as theory of competitive sports training is concerned, one of the important aims of developing explosive force training is to improve the basketball player's special force, which serves the purpose of directly improving the sport performance. The principle of specificity should be formed in the basketball player's explosive force training process, which requires that the movement range and direction as well as muscle group working nature and explosive force speed shall be mutually in conformity to the special skilled movement. This requires that the basketball player's movements like running, jumping and pitching should keep corresponding to the special skill as much as possible, so as to give play to the movement efficiency to the maximum limit.

\section{Notes in the basketball player's explosive force training}

First, load. When training the explosive force, the basketball players should not only actively develop their own agonistic muscle strength, but also pay attention to developing the strength of opposing muscle group and congener. Different loads will result in different effects. Therefore, the load should from person to person, and it is recommended to arrange the loads in a more reasonable 
way. In the training course, the basketball players should concentrate their attention in a better way to achieve a quick and instant explosive force.

Second, adjust training based on the actual situation. The exerciser should, according to the different requirements in different training stages, distinguish the progressively increasing explosive force combination strength and speed, etc. Thus, it can avoid the standstill in developing explosive force.

Third, enhance the special skill training. Enhancing the special skill training is able to improve the control force of muscle and its stretching and relaxing before exerting power, and other abilities. Once the relevant muscle groups are under tension status when exerting power, it will result in a not correct direction and angle of exerting power, therefore the distance of correctly exerting power skillfully will be shortened, and also the development of explosive force will be impacted.

Fourth, break between different sections of explosive force training. As a very high nervous energy will be consumed in the explosive force training, it is required to have a relatively long break time during the training, generally three to four minutes. In the training of improving explosive force, the most ideal effect is to depend on central nerve to generate a higher frequency towards motor unit. Therefore, it is required to actually guarantee the characteristics of explosive force, so as to avoid fatigue.

Fifth, there is a big difference in bounce between the basketball and other sports events. In the basketball competition, jumping with both feet accounts for over $80 \%$ in various jumping movements. Therefore, it is necessary to fully pay attention to the centripetal force contraction strength and speed of knee extensor and ankle extensor. In the meanwhile, it is recommended to intensify the development of exerciser's muscular eccentric contraction, improve the strength and extensibility of hamstrings, and moreover, the strength of upper limbs and body should be developed reasonably and the strength of lower limbs should be developed actively with the maximum weight. When developing the strength of exerciser's hamstrings, the overall speed force should be combined, to link the training of big muscle and small muscle, each single movement and several movements. The movements shall be guaranteed coordinated and coherent. The acting factors focusing on strength and speed need to be combined optimally. It is necessary to strictly control each basketball player's explosive force exercise intensity, times, sets, duration time and break time and other data, so that the basketball player can get the most ideal effect of explosive force training.

\section{Conclusion}

In conclusion, sufficient and effective basketball explosive force practice is able to obviously improve the basketball player's performance in special skills, and physical strength is the important foundation for basketball players to practically and effectively exercise, therefore making express power and explosive force become the important factors to improve the basketball player's performance in special skills. When working out the explosive force training plan, it is required to take a full consideration of the optimal combination of two acting factors including strength and speed. In the process of exerting explosive force, it is necessary, based on the characteristics of basketball sports, to train the corresponding skills according to the knowledge of exercise physiology and sports biochemistry. The training of explosive force shall be in different stages, to lay the foundation of maximum strength and further make the strength transform into explosive force. Therefore, it is an important guarantee for continuously improving the basketball player's performance in special skills through the optimal training of explosive force in the most reasonable way to greatly development the basketball player's explosive force.

\section{References}

[1] Peng Yanchun, Bi Xiushu: Research on Application of Basketball Player's Explosive Force Training Means, Sports Science, 2000 (June). 
[2] Li Shaohuai: Discussion on Lower limb’s Explosive Force Training of Basketball Players, Guizhou Sports Science and Technology, 2004 (January).

[3] Hu Xuechun: Study on Overload Training Method of Developing Muscular Power, Sichuan Sports Science, 2005 (February).

[4] Yi Zhiqiang: Training of Lower limb’s Muscular Explosive Force of Young Male Basketball Players, Journal of Science of Teachers’ College and University, 2007 (March).

[5] Ren Jianli: On Basketball Player's Explosive Force Training, Heilongjiang Science and Technology Information, 2011 (August). 